\title{
Telemedicine in dentistry as the gold standard of improving access to oral health care
}

Technology in the form of a smartphone and electronic media has penetrated into our lives no matter whether we live, in the country side or the city. There are numerous isolated villages and communities that do not have access to specialized medical care, the more so to dental care and prevention. Today, technology proposes a paradigm shift towards better interprofessional communication that helps both physicians and patients. Teledentistry is a combination of telecommunications and dentistry involving the exchange of clinical information and images over large distances for dental consultation and treatment planning.

Teledentistry is pertains to Telemedicine. Tele in Greek means distance and medicine in Latin means to heal. Teledentistry has been introduced in many branches of dentistry such as: endodontics, oral surgery, pedodontics, prosthetics, orthodontics, radiology, oral pathology. After the 2001 report on US telemedicine activity: the Association of Telehealth Service Providers has been highlighted by the yearly 2 -fold increase in the number of active telemedicine programs. This is justified by the development of telecommunications over the Internet [1]. Teledentistry was established in 1989 at the Westinghouse electronics system group conference in Baltimore; to be more specific, the birth of teledentistry as a specialist field of telemedicine could be linked to 1994 and a military project of the United States Army (US Army's Total Dental Access Project) [2].

In August 1996, Norway was the first European country to implement an official telemedicine levy program so that these services became reimbursable by the national health insurer [3].

Teledentistry has the role to eliminate the differences between oral health care in the urban and rural communities. by being quick, low cost and able to bring together specialists from different corners of the world in a very short time.

Teledentistry, also known as telemedicine in dentistry, will be included in the ADA's Code on Dental Procedures and Nomenclature CDT Code) for the first time in 2018 [4].

E-Oral Health technology that could address the participants's oral health needs in terms of education, consultation, screening, diagnosis, treatment, support or any other type of application in the field of dental medicine [2]. No limitation in terms of the duration of the intervention and the type of stakeholders that are involved in the interventions will be imposed.Teleconsultation through teledentistry can take place in either of the following ways - "Real-Time Consultation" and "Store-and Forward Method" [5].

Real-Time Consultation involves a videoconference in which dental professionals and their patients, at different locations, may see, hear, and communicate with one another.

Store-and-Forward Method involves the exchange of clinical information and static images collected and stored by the dental practitioner, who forwards them for consultation and treatment planning.

Teledentistry develops the ability to establish links between dental professionals to reduce the isolation phenomenon of rural and isolated areas in setting up a personalized treatment plan.

In the US, a software that facilitates teledentistry has been implemented. TeleDent by MouthWatch provides an easy, cost-effective, secure way to facilitate your health. It is designed to easily import x-ray pictures and digital scans for clinicians performing off-site dental examinations and to connect dental patients to imaging technology and clinical expertise at the point of care. It also tracks current CDT billing codes. With the advent of the new CDT 2018 codes, documentation will become even easier.

Despite being the only platform specifically designed for teledentistry, MouthWatch TeleDent is easy to implement and surprisingly affordable. The TeleDent subscription is available at an annual price of $\$ 299$ per provider for unlimited use [6].

Beside the software, implementing a teledentistry program requires adequate communication infrastructure, portable dental equipment, a source of sustainable funding, and a capable workforce trained to effectively use the equipment.

Teledentistry is also an educational tool that provides continuing education and training to dental professionals and others in dental schools and residency programs and in the clinics where it is used. The use of technology and teledentistry in education provides new opportunities for case learning and for the interactive participation in treatment planning and consultation from distant locations.

In a few years, most likely there will be legislation in each country that will support teledentistry. Awareness of teledentistry among health professionals should be increased and included in their day to day practice because it is a way to expand their practice, which can help in proper diagnosis and treatment plan accurately.

Recently, the UCLA School of Dentistry received a \$ 1.5 million grant from the largest dentistry provider to support the launch of a community-based clinical education program. The award will help UCLA student dentists to take care of the most vulnerable patients, as in California there is an uneven distribution of providers, underfunded public health programs, lack of understanding of the importance of oral health, and the difficulty of identifying available resources.

In honor of the World Oral Health Day (WOHD), FDI and Dentsply Sirona have signed a new partnership on endodontics. This collaboration will create a White Paper on endodontics and a Chairside Guide for dentists to be launched at the 2019 World Congress in San Francisco. To improve the quality of medical care of the readers of Stoma Edu J the use of teledentistry is strongly recommended.

Florin - Eugen Constantinescu DMD, PhD Student

Editorial Director, Product News

\section{References}

1. Sanchez Dils E, Lefebvre C, Abeyta K. Teledentistry in the United States: a new horizon of dental care. Int J Dent Hyg. 2004;2(4):161-164. DOI: 10.1111/j.16015037.2004.00093.x

[Full text links] [PubMed] Google Scholar (44) Scopus (15)

2. Marino R, Ghanim A. Teledentistry: a systematic review of the literature. J Telemed Telecare. 2013;19(4):179-183. doi: 10.1177/1357633X13479704. Review. [Full text links] [PubMed] Google Scholar (47) Scopus (23)

3. Hartvigsen $G$, Johansen MA, Hasvold $P$, et al. Challenges in telemedicine and eHealth: lessons learned from 20 years with telemedicine in Troms $\varnothing$. Stud Health Technol Inform. 2007;129(Pt 1):82-86

[Full text links] [PubMed] Google Scholar (39) Scopus (16)

4. Burger D. Code maintenance committee OKs teledentistry A1c codes. ADA News website. http://www.ada.org/ en/publications/ada-news/2017-archive/march/codemaintenance-committee-oks-teledentistry-a1c-codes Published March 14, 2017. Accessed March 20, 2017

5. Reddy KV. Using teledentistry for providing the specialist access to rural indians. Indian J Dent Res. 2011;22(2):189. doi: 10.4103/0970-9290.84275.

[Full text links] [Free full text] [PubMed] Google Scholar (20) Scopus (3)

6. Introducing TeleDent. MouthWatch website. https://www. mymouthwatch.com/teledentistry.php. Accessed March $27,2017$.

DOI: 10.25241/stomaeduj.2018.5(1).prodnews.1 